\title{
Home Sick with Coronavirus Symptoms: a National Study, April-May 2020
}

J Gen Intern Med 35(11):3409-12 DOI: $10.1007 / \mathrm{s} 11606-020-06159-5$

(c) Society of General Internal Medicine 2020

\section{INTRODUCTION}

Reports from hospitals and locales ${ }^{1}$ have highlighted racial/ethnic disparities in COVID-19 outcomes. However, few national studies in the USA have examined disparities among persons with symptoms suggestive of COVID-19. We analyzed a unique, nationally representative survey to assess demographic characteristics and social vulnerabilities among those with symptoms attributed to the coronavirus.

\section{METHODS}

We analyzed data on adults age 18-64 in weeks 1 (April 23-May 5) and 4 (May 21-26) of the Census Bureau's Household Pulse Survey (HPS), which collects data on pandemic-related health and financial problems. ${ }^{2}$ Individuals reporting not working the preceding week were asked why; one of 12 response options was "... because I am/ was sick with coronavirus symptoms." (Previous research demonstrated that $83.0 \%$ of Americans correctly identify fever, cough, and dyspnea as the three leading COVID-19 symptoms). ${ }^{3}$ We compared the demographic characteristics, health insurance status, food insecurity, and prevalence of COVID-19 in their state of residence ${ }^{4}$ (divided into quartiles), of those selecting this response to two other groups: (1) those working and (2) persons out-ofwork because of a non-coronavirus-related illness/ disability.

We performed univariate logistic regressions to evaluate the association of each factor with being out-sick due to coronavirus symptoms relative to each comparator group. We generated nationally representative estimates (and standard errors) using HPS' sample weights (and replicate weights) and Stata/ SE 16.1.

Received July 24, 2020

Accepted August 14, 2020

Published online September 10, 2020

\section{RESULTS}

Our sample included 89,490 adults working the past week, 457 out-sick with coronavirus symptoms, and 3503 out-ofwork because of a non-coronavirus illness/disability.

During May 21-26, 1.3 million workers nationally were out-sick because of coronavirus symptoms. Table 1 presents characteristics of that group and the comparator groups. Relative to both other groups, those out-sick with coronavirus symptoms were younger and more likely to be people of color: $24.2 \%$ were Black (vs. $11.5 \%$ of those working and $18.2 \%$ of those not-working due to a non-coronavirus illness/disability), $11.7 \%$ were Asian (vs. $5.6 \%$ and $2.0 \%$ ), and $26.5 \%$ were Hispanic (vs. $17.2 \%$ and $12.7 \%$ ).

Those out-sick with coronavirus symptoms were lesseducated and had lower incomes than those working; their education levels and incomes were more similar to those not working due to a non-coronavirus illness/disability. They had larger household size: $42.4 \%$ lived in a household with 5+ members, vs. less than a quarter in each comparison group.

$29.2 \%$ of those out-sick with coronavirus symptoms were uninsured, vs. $8 \%$ in each comparison group; $36.2 \%$ were food insecure, vs. $7.5 \%$ among those working and $20.8 \%$ among those out-sick for non-coronavirus symptoms/disability.

Finally, we observed an association between states' prevalence of coronavirus and being out-sick with coronavirus symptoms: $45.1 \%$ of Americans out-sick with coronavirus symptoms resided in a state in the top quartile of coronavirus prevalence, while only $15.2 \%$ lived in a bottom-quartile state. In contrast, individuals in the two comparison groups were divided roughly equally among the four state quartiles.

\section{DISCUSSION}

Minority race/ethnicity, low income, and residence in a state with high COVID prevalence were associated with work absence because of coronavirus symptoms in April-May 2020. This national-level evidence of the disparate impact of COVID-19 bolsters reports based on diagnoses from regions and hospital systems, ${ }^{1}$ as well as our previous findings of an increase in illness-related work absence in April that disproportionately affected minorities. ${ }^{5}$

We also identified social vulnerabilities - uninsurance and food insecurity - among many out-sick with coronavirus 


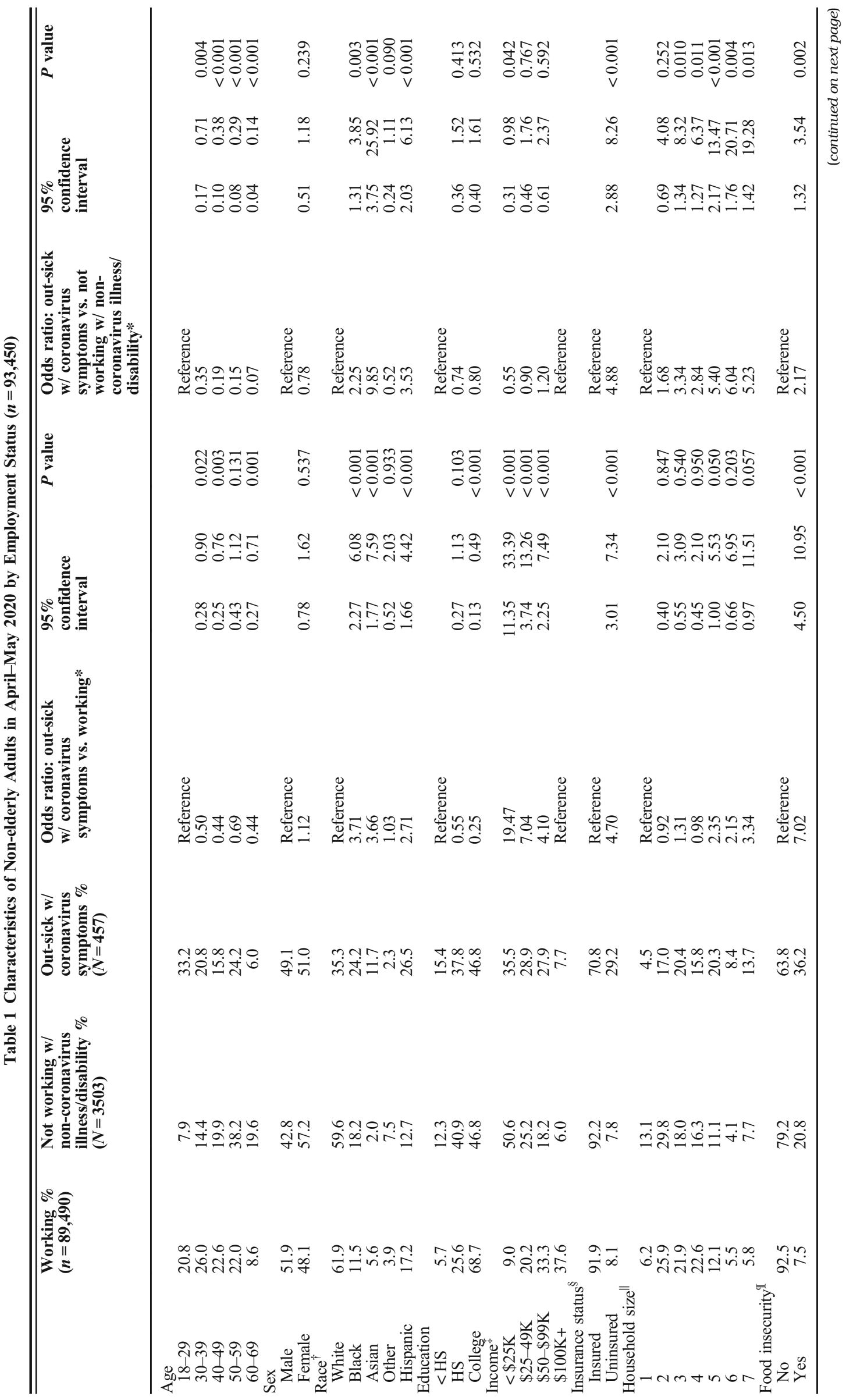




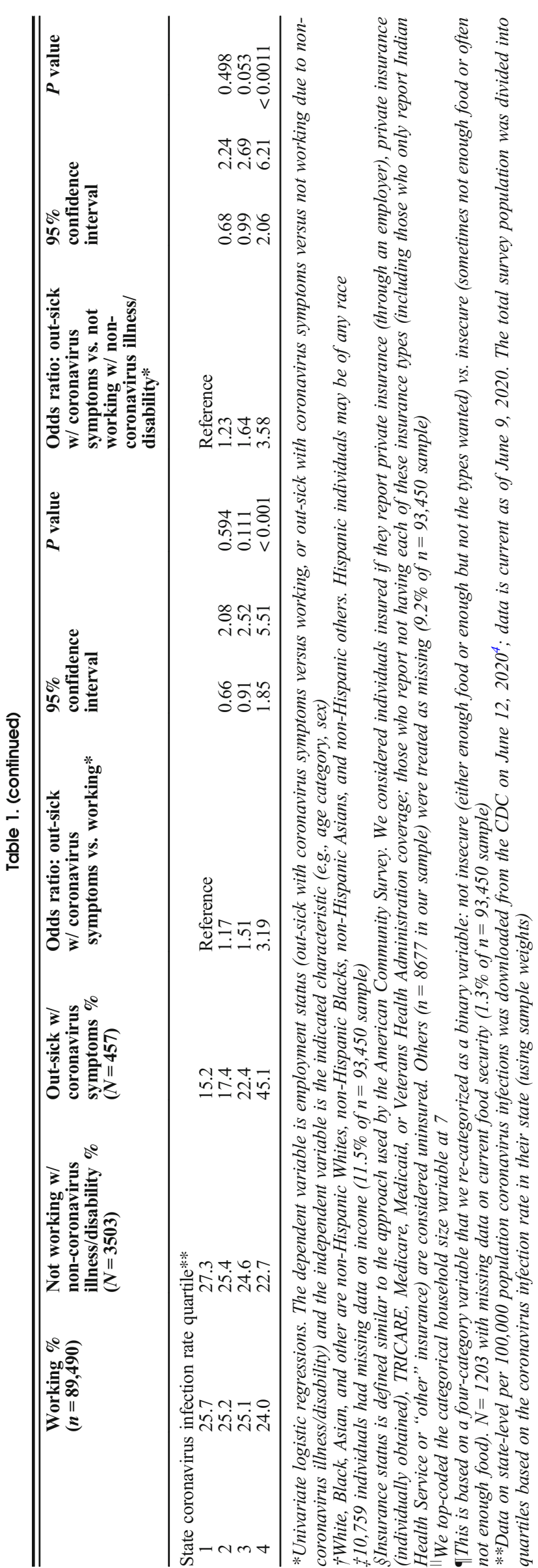

symptoms, which likely intensifies their risk of health and financial harms.

Our study has strengths and limitations. The Census Bureau recruited respondents via email and text messages to generate almost-real-time data; ${ }^{6}$ the trade-off was poorer response rates, ${ }^{2}$ which, despite weighting designed to account for nonresponse, may compromise generalizability. Additionally, the survey did not involve performance of diagnostic testing; some of those with "coronavirus symptoms" no doubt had other illnesses. However, the correlation between the statelevel COVID-19 infection rate and the proportion out-sick with coronavirus symptoms is reassuring, as is the consistency of findings across the two comparison groups. The high rate of uninsurance among those with "coronavirus symptoms" that we observed could, of course, obstruct medical evaluation and other care even among those with other illnesses.

During the COVID-19 pandemic, poor and minority Americans have been doubly disadvantaged: they are more often infected, but have fewer household resources and inferior health protection. Protecting the health and welfare of these patients must be a policy priority.

Adam W. Gaffney, $M D, M P H^{1,2}$

David Himmelstein, $M D^{2,3}$

David Bor, $M D^{1,2}$

Danny McCormick, $M D^{1,2}$

Steffie Woolhandler, $M D, M P H^{2,3}$

${ }^{1}$ Cambridge Health Alliance,

Cambridge, MA, USA

${ }^{2}$ Harvard Medical School,

Boston, MA, USA

${ }^{3}$ City University of New York at Hunter College,

New York, NY, USA

Corresponding Author: Adam W. Gaffney, MD, MPH; Cambridge Health Alliance, Cambridge, MA, USA (e-mail: agaffney@challiance.org).

\section{Compliance with Ethical Standards:}

Conflict of Interest: The authors report no financial conflicts of interest. Adam Gaffney, David Himmelstein, Steffie Woolhandler, and Danny McCormick serve as leaders of Physicians for a National Health Program (PNHP), a non-profit organization that favors coverage expansion through a single-payer program; however, none of them received any compensation from that group, although some of Dr. Gaffney's travel on behalf of the organization is reimbursed by it. David Bor is a member of PNHP.

\section{REFERENCES}

1. Azar KMJ, Shen Z, Romanelli RJ, et al. Disparities In Outcomes Among COVID-19 Patients In A Large Health Care System In California. Health Aff 2020; https://doi.org/10.1377/hlthaff.2020.00598.

2. US Census. Source of the Data and Accuracy of the Estimates for the 2020 Household Pulse Survey. [cited 2020 Jun 15]; Available from: https://www2. census.gov/programs-surveys/demo/technical-documentation/hhp/Sourceand-Accuracy-Statement-May-28-June2.pdf. Accessed 15 June 2020.

3. Alsan M, Stantcheva S, Yang D, Cutler D. Disparities in Coronavirus 2019 Reported Incidence, Knowledge, and Behavior Among US Adults. JAMA Netw Open 2020;3(6):e2012403. 
4. United States COVID-19 Cases and Deaths by State. CDC COVID Data Tracker [Internet] [cited 2020 Jun 12];Available from: https://www.cdc. gov/covid-data-tracker/index.html\#cases. Accessed 15 June 2020.

5. Gaffney A, Himmelstein D, Woolhandler S. Illness-related Work Absence in mid-April was Highest on Record. JAMA Intern Med 2020;In Press.
6. US Census Bureau. Measuring Household Experiences during the Coronavirus (COVID-19) Pandemic [Internet]. Census.gov. Available from: https://www.census.gov/householdpulsedata. Accessed 21 July 2020

Publisher's Note: Springer Nature remains neutral with regard to jurisdictional claims in published maps and institutional affiliations. 